\title{
Comparison of Extracorporeal Shock Wave Therapy and Trigger Point Injection in Terms of Their Effects on Pain and Bodily Functions of Myofascial Pain Syndrome Patients
}

\author{
Jung-Ho Lee, MSc ${ }^{1)}$, SAng-Hun JAng, MSc ${ }^{1)}$, Sung-Hyoun Cho, MSc ${ }^{1)}$, Jin-SAng Kim, PhD ${ }^{2)}$ \\ 1) Department of Physical Therapy, Daegu University Graduate School of Physical Therapy, Daegu \\ University: Naeri-ri, Jinlyang, Gyeongsan-si, Kyeongsangbuk-do, 15 Republic of Korea. TEL: +82 \\ 10-2934-5433, E-mail: upsh22@hanmail.net \\ 2) Department of Physical Therapy, Daegu College
}

\begin{abstract}
Purpose] This study examined how the non-invasive method of extracorporeal shock wave therapy (ESWT) and the invasive method of trigger point injection (TPI) affected subjects with myofascial pain syndrome (MPS) in the trapezius muscle in terms of alleviation pain and enhancement of bodily functions, and proposes a more effective treatment method. [Subjects] Thirty-one patients with MPS in the trapezius muscle were divided into three groups: an ESWT group ( $n=10)$, a TPI group $(n=13)$, and a control group $(n=8)$. [Methods] The degree of pain was measured using a visual analog scale (VAS). Pressure pain threshold (PPT) was measured at trigger points in the trapezius muscle using a pressure algometer, and the Constant-Murley scale (CMS) was used for functional evaluation. [Results] All three groups showed statistically significant improvements in terms of VAS following treatment. For the pressure pain threshold, only the TPI group showed a statistically significant improvement. CMS showed statistically significant improvements in all three groups, following the treatment. A comparison of the effects of the different treatment methods revealed no significant differences among the groups in terms of VAS and PPT, but CMS showed significant differences between the control and treatment groups. However, between the ESWT group and the TPI group, no significant difference was found in any category. [Conclusion] The study results suggest that the non-invasive method of ESWT is as effective as the invasive method of TPI in the treatment of myofascial pain syndrome.
\end{abstract}

Key words: Extracorporeal shock wave therapy, Trigger point injection, Myofascial pain syndrome

(This article was submitted May 15, 2012, and was accepted Jun. 11, 2012)

\section{INTRODUCTION}

In contemporary society, workers are spending increasing amounts of time sitting rather than standing, using the upper limbs more intensively than the lower limbs, and often suffer from pain and fatigue in the musculoskeletal system. Musculoskeletal diseases account for the largest share of functional diseases among people of working age and cause various problems for people in other age groups as well ${ }^{1)}$. Especially, neck and shoulder pain are very common. Fricton et al. examined 165 patients with chronic neck pain and headache and reported that myofascial pain syndrome was a primary cause for $55 \%$ of them ${ }^{2}$.

Myofascial pain syndrome is a very common type of musculoskeletal disease, and it is known to cause chronic pain $^{1)}$. In addition, it causes local pressure pain, taut band, spasm, and radial pain under pressure; it is defined as a local pain syndrome with particular pain trigger points. Its treatment methods include the relief of pain trigger points, the restoration of contracted muscles, and eliminating the causes of myofascial pain. More specifically, non-invasive methods include massage, stretching, physical training, drug treatment, and physical treatment, or trigger point injection (TPI) can be used as an invasive method ${ }^{3}$.

Extracorporeal shock wave therapy (ESWT) was first used in 1976 to resolve calculus in the kidneys and the bile duct. Since the early 1990s, the therapy has been broadly practiced in Germany for the treatment of various orthopedic diseases. As a non-invasive method, ESWT emits a shock wave which destroys damaged tissues and initiates recovery in the damaged parts by facilitating the formation of new blood vessels and stimulating local growth factors ${ }^{4)}$. ESWT alleviates pain, promotes the re-formation of local blood vessels and cell regeneration, and is widely used to treat the non-union of broken metacarpals, calcific tendinitis of the shoulder, plantar fasciitis, calcaneal spur, and patella syndrome. Several studies have reported that the therapy is also effective in the treatment of lateral epicondylitis ${ }^{5}$.

Trigger point injection (TPI) is known to be effective in the treatment of acute myofascial pain syndrome. It is an invasive method that performs injections at points that trigger pain due to muscle contraction or spasm. The injection 
1070 J. Phys. Ther. Sci. Vol. 24, No. 10, 2012

Table 1. General characteristics of subjects (Mean \pm SD)

\begin{tabular}{lccc}
\hline Variables & ESWT $(\mathrm{n}=10)$ & TPI $(\mathrm{n}=13)$ & SE $(\mathrm{n}=8)$ \\
\hline Age $(\mathrm{yr})$ & $48.2 \pm 12.0$ & $47.9 \pm 7.8$ & $46.5 \pm 13.1$ \\
Height $(\mathrm{cm})$ & $157.6 \pm 7.1$ & $156.9 \pm 4.0$ & $160.1 \pm 8.4$ \\
Weight $(\mathrm{kg})$ & $54.4 \pm 9.0$ & $52.7 \pm 3.5$ & $53.8 \pm 7.7$ \\
Gender (Male / Female) & $1 / 9$ & $1 / 12$ & $2 / 6$ \\
Affected side (Right / Left) & $7 / 3$ & $8 / 5$ & $6 / 2$ \\
Headache (Yes / No) & $1 / 9$ & $1 / 12$ & $1 / 7$ \\
\hline
\end{tabular}

ESWTG $=$ extracorporeal shock wave therapy, TIP $=$ trigger point injection, $\mathrm{SE}=$ stability exercise

methods vary: Travell's method injects $1-2 \mathrm{~mL}$ of procaine or lidocaine in a $0.5 \%$ concentration at pain trigger points; and Fischer's method injects $2-12 \mathrm{~mL}$ of $0.5 \%$ procaine or $1 \%$ lidocaine at pain trigger points and along the entire taut band. Sometimes, only needles are inserted at pain trigger points for stimulation ${ }^{6}$.

Many studies have been undertaken on the treatment of myofascial pain syndrome, but only a few of them have compared the effectiveness of invasive and non-invasive methods. This study examined how the non-invasive method of ESWT and the invasive method of TPI affect the pain and bodily functions of MPS patients, and compared the results to propose a more effective treatment method.

\section{SUBJECTS AND METHODS}

\section{Subjects}

We examined patients, who were diagnosed with MPS in the trapezius muscle between March 2011 and April 2012 by a medical doctor at $\mathrm{C}$ rehabilitation hospital in Cheongju, Republic of Korea. For inclusion in the study, subjects had to satisfy three or more of four conditions of the Simons MPS test: limited ROM, taut band, trigger point and pressure pain. Twelve subjects were excluded: three for neurological disease in the neck or the thoracic vertebrae, one because of an operation on the shoulder joint, three for internal diseases, and five because of an operation or other treatments during the research period. In the end, 31 patients participated in the study. They were randomly allocated to three groups: an ESWT group $(n=10)$, a TPI group $(n=13)$, and the control group $(n=8)$. The first two groups performed a stabilization exercise after the treatment sessions, and the control group performed only the stabilization exercise. All subjects received a full explanation of the purpose of the study and consented to participation (Table 1).

\section{Methods}

For the ESWT group, JEST-2000 (Joen Medical, Republic of Korea) was used. A gun (head $17 \mathrm{~mm}$ ) was used, and a low power shock wave $(5 \mathrm{~Hz}$ ) was applied 800 times using an air cylinder method. For the TPI group, Travell's method was used. A medical doctor injected $0.2 \mathrm{~mL}$ of $0.5 \%$ lidocaine at pain trigger points ${ }^{6}$. For the stabilization exercise, subjects lay face-down, adducted the shoulder blade, lowered it, and rotated it in order to keep the earlobe and acromial process on the same horizontal level. The subject maintained the posture for 10 seconds, took a three-second break, and repeated this 10 times for one set. A total of three sets were performed, with a three-minute break between each set. The treatment was applied once a week for two weeks.

The outcomes were measured before the first treatment session and after the second treatment session. The pain in the trapezius muscle was measured on a $100-\mathrm{mm}$ visual analog scale (VAS). It is a simple method that is frequently used in clinical tests. Subjects indicate their degree of pain on the scale between 0 (none) and 100 (extremely painful) $\mathrm{mm}^{7}$ ) In order to examine the pressure pain threshold (PPT), Fisher's pressure algometer was used at the pain trigger points of the trapezius muscle ${ }^{8)}$.

To assess the function of the shoulder joint, a standardized clinical method, the Constant-Murley scale (CMS), was used. It evaluates the degree of pain, range of motion, performance of daily activities, and muscle power, to yield a total score on a 100-point scale. Thirty-five points are reserved for subjective evaluation and 65 for objective evaluation. The subjective items include the degree of pain (15 points) and the performance of daily activities (20 points); objective items include the range of motion (40 points for flexion, abduction, and external and internal rotations) and muscle power of the shoulder ( 25 points). Higher score indicate better performance ${ }^{9)}$.

To describe the general characteristics of the treatment groups, a statistical analysis was conducted. The paired t-test was performed to examine changes within each group before and after the treatment. For inter-group comparison, one-way ANOVA was used. For statistical analysis, SPSS version 12.0 was used and significance was accepted for values of $\alpha<0.05$.

\section{RESULTS}

Table 2 shows the characteristics of the trapezius muscle in terms of VAS, PPT, and CMS, both before and after the treatment. All groups showed statistically significant changes in VAS following treatment $(p<0.05)$, only the TPI group showed statistically significant changes in PPT $(p<0.05)$. All three groups showed statistically significant changes in CMS $(p<0.05)$. 
Table 2. Comparison of VAS, PPT, and CMS between the pre-test and post-test in each group (Mean $\pm \mathrm{SD}$ )

\begin{tabular}{cccc}
\hline \multicolumn{2}{c}{ Group } & Pre-test & Post-test \\
\hline \multirow{2}{*}{ ESWT } & VAS* & $7.6 \pm 0.7$ & $4.4 \pm 1.4$ \\
$(\mathrm{n}=10)$ & PPT & $30.6 \pm 11.8$ & $35.0 \pm 13.7$ \\
& CMS* $^{*}$ & $57.8 \pm 7.1$ & $78.9 \pm 9.4$ \\
TPI & VAS $^{*}$ & $7.4 \pm 0.8$ & $4.2 \pm 1.2$ \\
$(\mathrm{n}=13)$ & PPT $^{*}$ & $31.2 \pm 8.3$ & $35.6 \pm 10.5$ \\
& CMS $^{*}$ & $60.2 \pm 6.5$ & $81.1 \pm 8.5$ \\
SE & VAS $^{*}$ & $7.3 \pm 1.8$ & $4.5 \pm 1.9$ \\
$(\mathrm{n}=8)$ & PPT & $29.8 \pm 12.1$ & $32.1 \pm 9.4$ \\
& CMS* $^{*}$ & $59.5 \pm 12.5$ & $72.9 \pm 13.9$ \\
\hline
\end{tabular}

$* \mathrm{p}<0.05$, VAS $=$ visual analog scale, PPT $=$ pressure pain threshold, $\mathrm{CMS}=$ Constant - Murley scale

Table 3 shows the results of the comparison among the different treatment methods. In terms of VAS and PPT, there were no significant differences among the groups, but CMS showed statistically significant differences among the groups $(\mathrm{p}<0.05)$. The results of the post-hoc test using LSD and Duncan showed that the treatment effects were significantly different between the ESWT group and the control group and between the TPI group and the control group $(p<0.05)$. However, between the ESWT group and the TPI group, there were no significant differences in any category.

\section{DISCUSSION}

Myofascial pain syndrome is characterized by an oversensitive response at trigger points, and the symptoms include pain, enervated muscles, limited range of motion, imbalance in the autonomous nervous system, depression, and sleep disturbance. The trigger points cause local or radial pain and accompany chronic musculoskeletal diseases ${ }^{10)}$.

Recent studies have reported that ESWT can be effective in the treatment of pain and enhances bodily functions ${ }^{11,12)}$. It is receiving heightened attention as an alternative treatment method for patients with musculoskeletal diseases, and related treatments are increasing ${ }^{13,14)}$. In this study, among the three therapy groups, the ESWT group showed significant improvements in VAS, which fell from $7.55 \pm$ 0.69 to $4.40 \pm 1.35$ following the treatment $(\mathrm{p}<0.05)$. CMS also improved significantly, increasing from $57.80 \pm 7.08$ to $78.90 \pm 9.41$ after the shock wave therapy $(p<0.05)$. These results are in agreement with the study of Furia, who examined 34 patients for the effects of ESWT on pain and bodily functions ${ }^{15)}$. Similarly, Hsu et al. treated 46 patients with ESWT and found significant changes in VAS and CMS $(p<0.05)^{16)}$. Haake et al. reported that some patients complained of slight increases in pain after ESWT, however the reaction was temporary ${ }^{17)}$.

TPI is widely used to treat MPS, but its effects are controversial. Ferrante et al. compared the effects of TPI and a placebo and reported no statistically significant difference ${ }^{18)}$. $\mathrm{Ga}$ et al. compared the effects of intramuscular stimulation (IMS) and TPI, and reported that the former was better at
Table 3. Comparison of effects among the groups (Mean \pm SD)

\begin{tabular}{cccr}
\hline & ESWT $(\mathrm{n}=10)$ & TPI $(\mathrm{n}=13)$ & SE $(\mathrm{n}=8)$ \\
\hline VAS & $3.2 \pm 0.9$ & $3.3 \pm 1.1$ & $2.8 \pm 0.9$ \\
PPT & $-4.5 \pm 9.1$ & $-4.5 \pm 9.4$ & $-2.2 \pm 7.4$ \\
CMS* & $-21.1 \pm 4.5$ & $-20.9 \pm 4.0$ & $-13.4 \pm 3.0$ \\
\hline
\end{tabular}

$* \mathrm{p}<0.05, \mathrm{VAS}=$ visual analog scale, $\mathrm{PPT}=$ pressure pain threshold,

$\mathrm{CMS}=$ Constant - Murley scale

alleviating pain and increasing the range of motion in the neck joint ${ }^{19)}$. The results of the present study show that the TPI group showed statistically significant improvements in VAS, PPT, and CMS after the treatment. This agrees with the study of Kim et al. who reported that TPI can be effective at lessening pain and enhancing range of motion ${ }^{20)}$.

A comparison of different treatment methods revealed no significant differences in terms of VAS and PPT. However, significant differences were found between the ESWT group and the control group and between the TPI group and the control group for CMS. Compared to the patients in the control group, which only performed a stabilization exercise, patients in other two groups showed greater improvements in bodily functions. This suggests that in the treatment of MPS, combination therapies may be more effective than applying only one treatment method ${ }^{21)}$.

The comparison of VAS, PPT, and CMS found no significant treatment difference between the ESWT group and the TPI group. In other words, in terms of alleviating pain, reducing the pressure pain threshold and improving bodily function, the invasive method of TPI and the non-invasive method of ESWT were equally effective. However, there could have been a bias in this study due to the small number of subjects, the short of therapy period, and the fact that the patients in all three groups performed a stabilization exercise, possibly over-evaluating the effect of the treatment.

\section{ACKNOWLEDGEMENT}

This research was supported (in part) by the Deagu University Research Grant, 2008.

\section{REFERENCES}

1) Cole TM, Edgerton VR: Report of the task force on medical rehabilitation research, Hunt Valley: National Institute of Health, 1990, pp 61-70.

2) Fricton JR, Auvinen MD, Dykstra D, et al.: Myofascial pain syndrome: electromyographic changes associated with local twitch response. Arch Phys Med Rehabil, 1985, 66: 314-317. [Medline]

3) Gerwin RD: Classification, epidemiology, and natural history of myofascial pain syndrome. Curr Pain Headache Rep, 2001, 5: 412-420. [Medline] [CrossRef]

4) Speed CA, Nichols D, Richards C, et al.: Extracorporeal shock wave therapy for lateral epicondylitis - a double blind randomized controlled trial. J Orthop Res, 2006, 20: 895-898. [CrossRef]

5) Haupt G: Use of extracorporeal shock waves in the treatment of pseudarthrosis, tendinopathy and other orthopedic diseases. J Urol, 1997, 158: 4-11. [Medline] [CrossRef]

6) Travell JG, Simons DG: Myofascial pain and dysfunction. The trigger point manual. Baltimore: Williams and Willans, 1999, pp11-71.

7) DeLoach LJ, Higgins MS, Caplan AB, et al.: The visual analog scale in the immediate postoperative period: Intrasubject variability and correlation with a numeric scale. Anesth Analg, 1998, 86: 102-106. [Medline] 
[CrossRef]

8) Fischer AA: Pressure threshold meter: Its use for quantification of tender spots. Arch Phys Med Rehabil, 1986, 67: 836-838. [Medline]

9) Constant CR, Murley AH: A clinical method of functional assessment of the shoulder. Clin Orthop Relat Res, 1987, 214: 160-164. [Medline]

10) Alvarez DJ, Rockwell PG: Trigger points: diagnosis and management. Am Fam Physician, 2002, 65: 653-661. [Medline]

11) Oh JH, Lhee SH, Park JY, et al.: Extracorporeal shock wave therapy versus platelet-rich plasma injection for the treatment of lateral epicondylitis: a prospective randomized clinical trial. J Korean Soc Surg Hand, 2011, 16: 241-246.

12) Furia JP, Rompe JD, Maffulli N: Low-energy extracorporeal shock wave therapy as a treatment for greater trochanteric pain syndrome. Am J Sports Med, 2009, 37: 1806-1813. [Medline] [CrossRef]

13) Seil R, Wilmes P, Nührenbörger C: Extracorporeal shock wave therapy for tendinopathies. Expert Rev Med Devices, 2006, 3: 463-470. [Medline] [CrossRef]

14) Kuo YR, Wang CT, Wang FS, et al.: Extracorporeal shock-wave therapy enhanced wound healing via increasing topical blood perfusion and tissue regeneration in a rat model of STZ-induced diabetes. Wound Repair Regen, 2009, 17: 522-530. [Medline] [CrossRef]

15) Furia JP: High-energy extracorporeal shock wave therapy as a treatment for chronic noninsertional achilles tendinopathy. Am J Sports Med, 2008, 36: 502-508. [Medline] [CrossRef]

16) Hsu CJ, Wang DY, Tseng KF, et al.: Extracorporeal shock wave therapy for calcifying tendinitis of the shoulder. J Shoulder Elbow Surg, 2008, 17: 55-59. [Medline] [CrossRef]

17) Haake M, Boddeker IR, Decker T, et al.: Side-effects of extracorporeal shock wave therapy (ESWT) in the treatment of tennis elbow. Arch Orthop Trauma Surg, 2002, 122: 222-228. [Medline] [CrossRef]

18) Ferrante FM, Bearn L, Rothrock R, et al.: Evidence against trigger point injection technique for the treatment of cervicothoracic myofascial pain with botulinum toxin type A. Anesthesiology, 2005, 103: 377-383. [Medline] [CrossRef]

19) Ga H, Koh HJ, Choi JH, et al.: Intramuscular and nerve root stimulation vs lidocaine injection of trigger points in myofascial pain syndrome. J Rehabil Med, 2007, 39: 374-378. [Medline] [CrossRef]

20) Kim GU, Choe SC, Song HJ, et al.: Trigger point injection for the treatment of cervical strain in the emergency department. Korean Soc Traumatol, 2002, 15: 33-38.

21) Fumich RM, Ellison AE, Guerin GJ, et al.: The measured effect of taping on combined foot and ankle motion before and after exercise. Am J Sports Med, 1981, 9: 165-170. [Medline] [CrossRef] 